\title{
SPIN-ORBIT ALIGNMENT FOR THE CIRCUMBINARY PLANET HOST KEPLER-16 A
}

\author{
Joshua N. Winn ${ }^{1}$, Simon Albrecht ${ }^{1}$, John Asher Johnson ${ }^{2,3}$, Guillermo Torres ${ }^{4}$, William D. Cochran ${ }^{5}$, \\ Geoffrey W. Marcy ${ }^{6}$, ANdrew W. Howard ${ }^{6}$, Howard IsaACSON ${ }^{6}$, Debra Fischer ${ }^{7}$, Laurance Doyle ${ }^{8}$, William Welsh ${ }^{9}$, \\ Joshua A. Carter ${ }^{4}$, Daniel C. Fabrycky ${ }^{10,19}$, Darin Ragozzine ${ }^{4}$, SAmuel N. Quinn $^{4}$, Avi ShPorer ${ }^{11}$, Steve B. Howell ${ }^{12}$, \\ David W. Latham ${ }^{4}$, Jerome Orosz ${ }^{9}$, Andrej Prsa ${ }^{13}$, Robert W. Slawson ${ }^{8}$, William J. Borucki ${ }^{14}$, David Koch ${ }^{14}$, \\ Thomas Barclay ${ }^{15}$, Alan P. Boss ${ }^{16}$, Jørgen Christensen-DalsGaArd ${ }^{17}$, Forrest R. Girouard ${ }^{14,18}$, Jon Jenkins ${ }^{8,14}$, \\ Todd C. Klaus ${ }^{18}$, Søren Meibom ${ }^{4}$, Robert L. Morris ${ }^{8,14}$, Dimitar Sasselov ${ }^{4}$, Martin Still ${ }^{15}$, and JefFrey Van Cleve ${ }^{8,14}$ \\ ${ }^{1}$ Department of Physics, and Kavli Institute for Astrophysics and Space Research, Massachusetts Institute of Technology, Cambridge, MA 02139, USA \\ ${ }^{2}$ Department of Astrophysics, California Institute of Technology, MC249-17, Pasadena, CA 91125, USA \\ ${ }^{3}$ NASA Exoplanet Science Institute (NExScI) \\ ${ }^{4}$ Harvard-Smithsonian Center for Astrophysics, Cambridge, MA 02138, USA \\ ${ }^{5}$ McDonald Observatory, The University of Texas, Austin, TX 78712, USA \\ ${ }^{6}$ Department of Astronomy, University of California, Berkeley, CA 94720, USA \\ ${ }^{7}$ Department of Astronomy, Yale University, New Haven, CT 06511, USA \\ ${ }^{8}$ Carl Sagan Center for the Study of Life in the Universe, SETI Institute, Mountain View, CA 94043, USA \\ ${ }^{9}$ Astronomy Department, San Diego State University, San Diego, CA 92182, USA \\ ${ }^{10}$ Department of Astronomy and Astrophysics, University of California, Santa Cruz, CA 95064, USA \\ ${ }^{11}$ Las Cumbres Observatory Global Telescope Network, Santa Barbara, CA 93117, USA \\ ${ }^{12}$ National Optical Astronomy Observatory, Tucson, AZ 85726, USA \\ ${ }^{13}$ Department of Astronomy and Astrophysics, Villanova University, Villanova, PA 19085, USA \\ ${ }^{14}$ NASA Ames Research Center, Moffett Field, CA 94035, USA \\ ${ }^{15}$ Bay Area Environmental Research Institute/NASA Ames Research Center, Moffett Field, CA 94035, USA \\ ${ }^{16}$ Department of Terrestrial Magnetism, Carnegie Institution of Washington, Washington, DC 20015, USA \\ 17 Danish AsteroSeismology Centre, and Department of Physics and Astronomy, Aarhus University, Ny Munkegade, DK-8000 Aarhus C, Denmark \\ ${ }^{18}$ Orbital Sciences Corporation/NASA Ames Research Center, Moffett Field, CA 94035, USA \\ Received 2011 August 3; accepted 2011 September 21; published 2011 October 5
}

\begin{abstract}
Kepler-16 is an eccentric low-mass eclipsing binary with a circumbinary transiting planet. Here, we investigate the angular momentum of the primary star, based on Kepler photometry and Keck spectroscopy. The primary star's rotation period is $35.1 \pm 1.0$ days, and its projected obliquity with respect to the stellar binary orbit is $1.6 \pm 2.4$. Therefore, the three largest sources of angular momentum - the stellar orbit, the planetary orbit, and the primary's rotation-are all closely aligned. This finding supports a formation scenario involving accretion from a single disk. Alternatively, tides may have realigned the stars despite their relatively wide separation (0.2 AU), a hypothesis that is supported by the agreement between the measured rotation period and the "pseudosynchronous" period of tidal evolution theory. The rotation period, chromospheric activity level, and fractional light variations suggest a main-sequence age of 2-4 Gyr. Evolutionary models of low-mass stars can match the observed masses and radii of the primary and secondary stars to within about $3 \%$.
\end{abstract}

Key words: binaries: general - planets and satellites: formation - stars: individual (Kepler-16 A, KIC 12644769) stars: low-mass - stars: rotation

Online-only material: color figures

\section{INTRODUCTION}

Kepler-16 (AB)-b is a planet with two parent stars (Doyle et al. 2011). The stars $\left(0.7\right.$ and $\left.0.2 M_{\odot}\right)$ are in a 41 day eccentric orbit, and the planet $\left(0.3 M_{\mathrm{Jup}}\right)$ circles both of them every 229 days. Viewed from the solar system, the stars eclipse each other and the planet transits both of them, providing definitive evidence that circumbinary planets exist and permitting precise determinations of the system's parameters. For example, the planet's radius is known to within $0.3 \%$, better than that of any other known exoplanet. The stars are themselves of interest as a rare example of low-mass dwarfs with precisely known dimensions.

Such a unique system should be studied in every possible way, for exploratory purposes as well as the specific purpose of understanding its formation and evolution. How old are the stars? Did the planet form together with the stars, or was it

\footnotetext{
${ }^{19}$ Hubble Fellow.
}

captured from another system? Has there been tidal evolution or other effects that have modified the system's architecture? Here we present an investigation of the angular momentum of the primary star, bearing on these questions.

It has already been established that the planes of the circumbinary orbit and the stellar orbit are aligned to within 0.5 (Doyle et al. 2011). This suggests that all three bodies inherited their angular momentum from a single disk, as opposed to dynamical scenarios that are often invoked for triple systems such as close encounters (Mikkola 1984; Bailyn 1989; Ivanova 2008) or dynamical decay (Sterzik \& Tokovinin 2002). One must remember, though, that the planet was discovered with transit photometry, a technique that is severely biased toward finding coplanar orbits. This raises the question of whether the orbital coplanarity of Kepler-16 is at all representative of circumbinary planets, and motivates measurements of the alignment between the orbital axes and the stellar spin axes, for which there was no selection bias. 

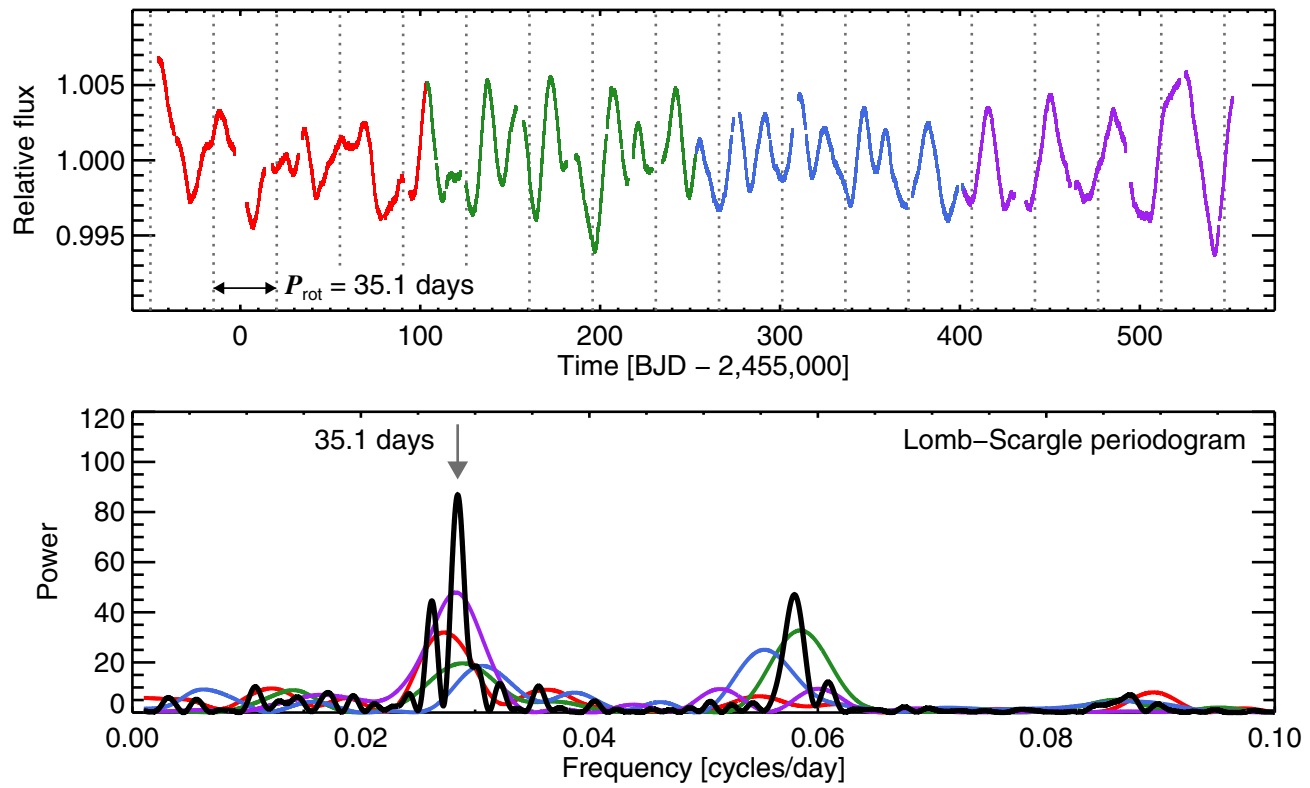

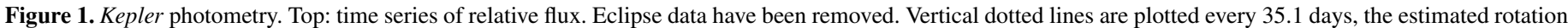

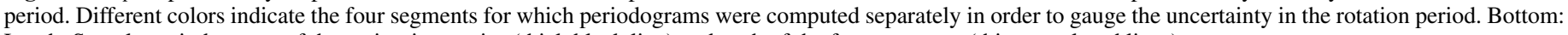
Lomb-Scargle periodograms of the entire time series (thick black line) and each of the four segments (thinner colored lines).

(A color version of this figure is available in the online journal.)

This Letter is organized as follows. Section 2 presents a photometric determination of the rotation period. Section 3 presents a spectroscopic determination of the sky-projected stellar obliquity (the angle between the rotation axis of the primary star and the stellar orbital axis), based on observations of the Rossiter-McLaughlin (RM) effect. Section 4 discusses the implications of these results for our understanding of the primary star and of the system's history.

\section{THE ROTATION PERIOD}

We measured the rotation period of the primary star with data from the Kepler spacecraft, a $0.95 \mathrm{~m}$ space telescope that monitors the optical brightness of about 150,000 stars in a quest to detect transits of potentially habitable Earth-sized planets (Borucki et al. 2010). Overviews of the mission design, the instrument performance, and the data processing pipeline were given by Koch et al. (2010), Caldwell et al. (2010), and Jenkins et al. (2010).

Kepler-16 was observed with a 29.4 minute sampling for a nearly continuous 600 day interval, from 2009 May 2 to 2010 December 22 (quarters 1-7). The duty cycle was $94 \%$, with 17 short gaps due to technical problems as well as scheduled interruptions in observing. After each interruption a jump was observed in the relative flux. We placed all the data onto a common flux scale under the assumption that the flux variations during the interruptions were smooth enough to be described by a quadratic function of time. Specifically, we multiplied the data from each of the 18 disjoint intervals by a constant and determined the optimal values of the constants by fitting quadratic functions to the data within 1 day of an interruption.

The resulting time series exhibited a secular $3 \%$ decrease in relative flux, which could be an instrumental effect or a true decrease in stellar brightness. Since this trend is irrelevant to the rotation period determination, we applied a 70 day median filter prior to plotting the time series in the top panel of Figure 1. The time series exhibits quasi-periodic variations of order $0.5 \%$. As usual for late-type stars, we attribute these variations to dark spots and bright plages being carried around by stellar rotation.

The bottom panel of Figure 1 is a Lomb-Scargle periodogram, showing a prominent peak at 35.1 days along with smaller peaks at the first two harmonics. We identified this peak with the stellar rotation period. We estimated the uncertainty in the period by dividing the data chronologically into four equal segments, analyzing each piece separately, and finding the standard deviation in the mean of the periodogram peaks. Based on this analysis we find $P_{\text {rot }}=35.1 \pm 1.0$ days.

\section{THE ROSSITER-MCLAUGHLIN EFFECT}

We measured the sky-projected obliquity and rotation rate of the primary star by conducting spectroscopic observations of a primary eclipse and analyzing the RM effect. The RM effect is the anomalous Doppler shift that is observed during eclipses as a consequence of the selective blockage of the rotating stellar photosphere (Rossiter 1924; McLaughlin 1924).

We used the Keck I 10 m telescope and High Resolution Echelle Spectrometer spectrograph to gather 14 spectra on 2011 May 28/29, starting 40 minutes before ingress and extending for $5 \mathrm{hr}$ until morning twilight, thereby covering about threequarters of the eclipse. Another three spectra were obtained the following night to track the out-of-eclipse velocity variation. The typical exposure time was 19 minutes. The $\mathrm{I}_{2}$ absorption cell was used to establish the wavelength scale and instrumental profile. A single exposure without $\mathrm{I}_{2}$ was also obtained to serve as a template spectrum. The relative radial velocities (RVs) were determined with a descendant of the algorithm of Butler et al. (1996). They are given in Table 1 and plotted in Figure 2.

The "red-then-blue" pattern of the anomalous Doppler shift is characteristic of a prograde orbit with good alignment between the primary star's rotational and orbital angular momenta. In the first half of the eclipse, the secondary covers the approaching (blue) half of the primary, causing the net starlight to be redshifted; then, the secondary moves over the receding (redshifted) half of the primary, producing an anomalous blueshift. 

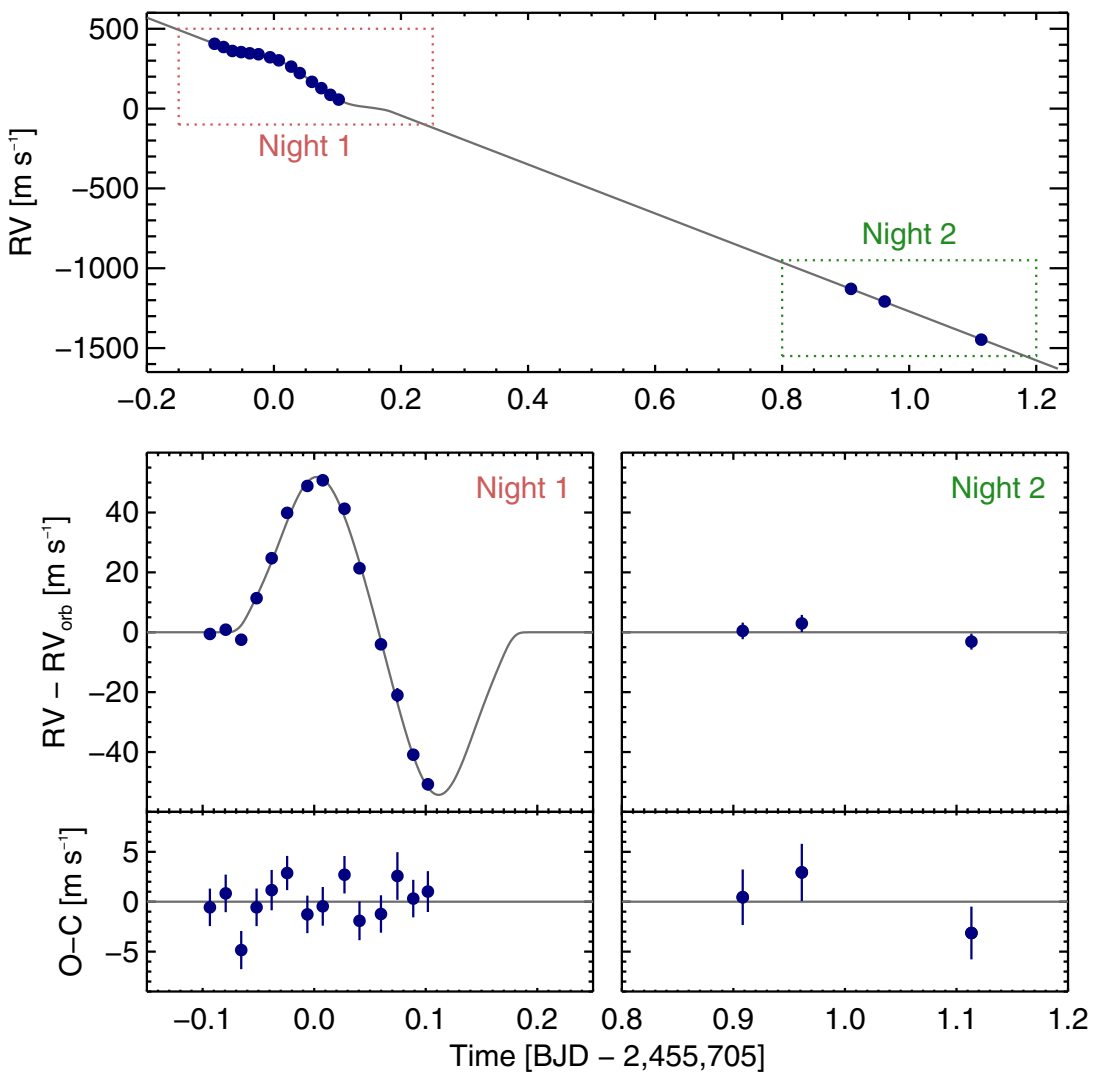

Figure 2. Keck radial velocities. Top: apparent radial velocity (solid points) and the best-fitting model (gray curve). Bottom: after subtracting the best-fitting orbital model, thereby isolating the RM anomaly. Each night's data are shown separately, along with the residuals.

(A color version of this figure is available in the online journal.)

Table 1

Relative Radial Velocity Measurements of Kepler-16 A

\begin{tabular}{lrc}
\hline \hline BJDUTC & $\begin{array}{c}\text { RV } \\
\left(\mathrm{m} \mathrm{s}^{-1}\right)\end{array}$ & $\begin{array}{c}\text { Unc. } \\
\left(\mathrm{m} \mathrm{s}^{-1}\right)\end{array}$ \\
\hline 2455704.90651 & 405.81 & 1.88 \\
2455704.92067 & 385.56 & 1.88 \\
2455704.93459 & 360.90 & 1.91 \\
2455704.94835 & 353.70 & 1.88 \\
2455704.96188 & 346.34 & 2.02 \\
2455704.97574 & 340.26 & 1.72 \\
2455704.99387 & 321.48 & 1.88 \\
2455705.00766 & 302.26 & 1.94 \\
2455705.02716 & 262.90 & 1.88 \\
2455705.04061 & 222.41 & 1.94 \\
2455705.05985 & 167.53 & 1.87 \\
2455705.07461 & 127.95 & 2.39 \\
2455705.08883 & 86.27 & 1.88 \\
2455705.10192 & 56.34 & 2.05 \\
2455705.90834 & -1129.19 & 2.78 \\
2455705.96133 & -1207.97 & 2.86 \\
2455706.11341 & -1447.21 & 2.64 \\
\hline
\end{tabular}

For quantitative modeling we used the technique of Albrecht et al. (2007), in which a pixellated stellar disk is constructed, and a theoretical spectral line profile is computed for each pixel based on the local intensity and velocity of the photosphere. The integrated spectrum is obtained by summing over the uneclipsed pixels, and then the RV is calculated by cross-correlation with the uneclipsed, disk-integrated spectrum.
To compute the relative intensities of the pixels, we assumed a linear limb-darkening law. The pixel velocities included the effects of uniform rotation, macroturbulence, and the convective blueshift. The model for macroturbulence was taken from Gray (2005), assuming equal radial and tangential velocity perturbations with a standard deviation $\zeta_{\mathrm{RT}}=2.5 \mathrm{~km} \mathrm{~s}^{-1}$. The model for the convective blueshift was taken from Shporer \& Brown (2011), in which the velocity of each pixel is shifted by $V_{\mathrm{CB}}=0.2 \mathrm{~km} \mathrm{~s}^{-1}$ away from the center of the star. The stellar radii and orbital inclination were held fixed at the values determined by Doyle et al. (2011). We neglected any light from the secondary, as the light ratio is constrained to be $<1.3 \%$ (Doyle et al. 2011).

The six adjustable parameters were the projected rotation rate $v \sin i$, the projected obliquity $\beta$, the limb darkening coefficient $u$, the central eclipse time $T_{c}$, the zero point of the relative RV scale $\gamma$, and a parameter describing the out-ofeclipse RV variation. For the latter, a simple linear slope would have sufficed, but for convenience we used $M_{B}^{\prime}$, the mass of the secondary when all other orbital parameters (period, inclination, eccentricity, argument of pericenter) are held fixed at the bestfitting values reported by Doyle et al. (2011). We expect small differences between $M_{B}^{\prime}$ and the true secondary mass $M_{B}$ due to the small uncertainties in the other orbital parameters as well as spurious radial accelerations caused by starspots or light from the secondary.

To determine the allowed parameter ranges we used a Markov Chain Monte Carlo algorithm, with the Metropolis-Hastings algorithm and Gibbs sampler. The likelihood was taken to be $\exp \left(-\chi^{2} / 2\right)$, where $\chi^{2}$ is the usual sum of the standardized 
Table 2

Summary of Results

\begin{tabular}{lc}
\hline \hline Parameter & Value \\
\hline Projected rotation speed, $v \sin i\left(\mathrm{~km} \mathrm{~s}^{-1}\right)$ & $0.920 \pm 0.025$ \\
Projected spin-orbit angle, $\beta(\mathrm{deg})$ & $1.6 \pm 2.4$ \\
Secondary mass parameter, $M_{B}^{\prime}\left(M_{\odot}\right)$ & $0.20133 \pm 0.00026$ \\
Velocity zero point, $\gamma\left(\mathrm{m} \mathrm{s}^{-1}\right)$ & $427.6 \pm 1.3$ \\
Limb darkening coefficient, $u$ & $0.717 \pm 0.079$ \\
Central eclipse time, $T_{c}(\mathrm{BJD}$ UTC $)$ & $2,455,705.05388 \pm 0.00069$ \\
\hline Stellar rotation period, $P_{\text {rot }}(\mathrm{days})$ & $35.1 \pm 1.0$ \\
Stellar inclination angle, $i(\mathrm{deg})$ & $90 \pm 9$ \\
Stellar obliquity, $\psi(\mathrm{deg})$ & $418.3(95.4 \% \mathrm{conf})$. \\
\hline Effective temperature, $T_{\text {eff }}(\mathrm{K})$ & $4337 \pm 80$ \\
Iron abundance, $[\mathrm{Fe} / \mathrm{H}]$ & $-0.04 \pm 0.08$ \\
Surface gravity, $\log \left(g\left(\mathrm{~cm} \mathrm{~s}{ }^{-2}\right)\right)$ & $4.6527 \pm 0.0017$ \\
Main-sequence age $(\mathrm{Gyr})$ & $3.0 \pm 1.0$ \\
\hline
\end{tabular}

Notes. All the stellar parameters refer to the primary star, Kepler-16 A, except for the secondary mass parameter $M_{B}^{\prime}$ (defined in the text). The result for $M_{B}^{\prime}$ differs by $0.00120 \pm 0.00071 M_{\odot}$ from the secondary mass $M_{B}=0.20255 \pm 0.00066 M_{\odot}$ determined by Doyle et al. (2011).

residuals between the observed and calculated RVs. Uniform priors were adopted for $v \sin i, \beta, \gamma$, and $M_{2}^{\prime}$. A Gaussian prior was used for $T_{c}$, with a central value equal to the predicted ephemeris time and a standard deviation of 1 minute (the typical level of eclipse timing variations). A Gaussian prior was used for the limb darkening coefficient, $u=0.8 \pm 0.1$, based on the tables of Claret (2000).

The formal " $1 \sigma$ " uncertainty interval was taken to be the range between the $15.8 \%$ and $84.2 \%$ levels of the cumulative distribution of the marginalized posterior for each parameter. In addition, we checked on the sensitivity of the results to the assumed values of the macroturbulent velocity $\zeta_{\mathrm{RT}}$ and convective blueshift $V_{\mathrm{CB}}$, by perturbing each of those quantities by $\pm 50 \%$ and refitting. (It would be better to allow these parameters to vary during the fit, but in practice this was too computationally demanding.) Changing the macroturbulence had no appreciable effect. Changing the convective blueshift caused shifts of $18 \mathrm{~m} \mathrm{~s}^{-1}$ in $v \sin i$ and $2^{\circ}$ in $\beta$, comparable to the formal $1 \sigma$ intervals. Consequently, we enlarged the uncertainty intervals for those two parameters by adding those shifts in quadrature with the formal errors. Table 2 gives the results for all the parameters.

\section{DISCUSSION}

\subsection{Spin-Orbit Alignment}

The angle $\beta$ between the sky projections of the primary's rotational angular momentum and the orbital angular momentum of the binary was found to be $1.6 \pm 2.4 .^{20}$ The star is apparently aligned with the orbit to within a few degrees.

Ordinarily a warning must be issued here: the stellar rotation axis might be inclined along the line of sight, and the skyprojected obliquity $\beta$ might not be representative of the true three-dimensional obliquity $\psi$. In this case, though, an upper bound on the true obliquity is enforced by the combination $v \sin i=0.920 \pm 0.025 \mathrm{~km} \mathrm{~s}^{-1}$ from the RM analysis, $P_{\mathrm{rot}}=$ $35.1 \pm 1.0$ days from the Kepler data, and $R_{\star}=0.6489 \pm$

\footnotetext{
20 The angle $\beta$ is defined using the coordinate system of Hosokawa (1953). Note that Ohta et al. (2005) and others have used a different symbol $\lambda$ to represent this angle, and a coordinate system such that $\lambda=-\beta$.
}

$0.0013 R_{\odot}$ from the photometric-dynamical model of Doyle et al. (2011). Assuming $v=2 \pi R_{\star} / P_{\text {rot }}$, and adopting a isotropic prior for $i$ (uniform in $\cos i$ ), we find $\sin i=0.994_{-0.043}^{+0.006}$ and $i=90^{\circ} \pm 9^{\circ}$. With $95.4 \%$ confidence, the true obliquity is $\psi<18.3$.

This system's angular momentum has five contributions- the stellar orbit, the planetary orbit, the primary rotation, the secondary rotation, and the planetary rotation-with magnitudes in the approximate ratios 10,000:40:1:0.1:0.001. Kepler-16 is an orderly system, with good alignment between the three largest portions of the angular momentum. To our knowledge, Kepler-16 is the longest-period stellar binary for which a stellar obliquity has been measured (see Table 1 of Albrecht et al. 2011).

\subsection{Activity, Rotation, and Age}

The Keck spectra show $\mathrm{Ca} H \& \mathrm{~K}$ chromospheric emission with $\log R_{\mathrm{HK}}^{\prime}=-4.68 \pm 0.10$, stronger than the Sun's value of -4.91. The spectra obtained previously by Doyle et al. (2011) also exhibit $\mathrm{Ca} H \& \mathrm{~K}$ emission, with $\mathrm{RV}$ variations tracking those of the primary, proving that the emission originates on the primary rather than the secondary or the planet. We may therefore use empirical relations between the rotation, chromospheric activity, and age of main-sequence dwarfs to estimate the age of Kepler-16 A and check whether there is anything unusual about its properties.

Building on work by Barnes (2007) and others, Mamajek \& Hillenbrand (2008) provided up-to-date activity/rotation/age relations for $\mathrm{K} 2-\mathrm{F} 7 \mathrm{dwarfs}\left(\approx 0.8-1.3 M_{\odot}\right)$, which we extrapolated to interpret the $0.7 M_{\odot}$ primary of Kepler-16. The activity/age relation in their Equation (3) gives an age of $1.8 \pm 1.2$ Gyr. The rotation/age relation implicit in their Equations (12)-(14) give an age of $3.7 \pm 0.8 \mathrm{Gyr}$. A similar age is obtained from the relations of Barnes (2010) and Barnes \& Kim (2010).

In addition, the rotation and activity are expected to be directly linked, with a particularly strong correlation between the Rossby number Ro (the ratio of rotation period to convective turnover timescale) and $\log R_{\mathrm{HK}}^{\prime}$. We used Equation (4) of Noyes et al. (1984) to estimate the convective turnover timescale, obtaining $\tau_{c}=23.0$ days and $\mathrm{Ro}=1.52$. The relation between Ro and chromospheric activity shown in Figure 7 of Mamajek \& Hillenbrand (2008) predicts $\log R_{\mathrm{HK}}^{\prime}=-4.70$, in good agreement with the measured value. The light variations of $0.5 \%-1 \%$ are also typical for a star with this level of chromospheric emission (see, e.g., Hall et al. 2009).

All together, the rotation period, chromospheric emission level, and fractional light variations paint a picture of an ordinary $0.7 M_{\odot}$ dwarf star with an age of 2-4 Gyr.

\subsection{Comparison to Evolutionary Models}

The Keck spectrum provides new estimates of the primary's photospheric parameters. Analysis with Spectroscopy Made Easy, a software package written by Valenti \& Piskunov (1996), gives $T_{\text {eff }}=4337 \pm 80 \mathrm{~K}$ and $[\mathrm{Fe} / \mathrm{H}]=-0.04 \pm 0.08$ for a fixed value of $\log g=4.6527$ (the value determined by Doyle et al. 2011, which has a negligible uncertainty for this purpose). These agree with the previously reported results $T_{\text {eff }}=4450 \pm 150 \mathrm{~K}$ and $[\mathrm{m} / \mathrm{H}]=-0.3 \pm 0.2$ (Doyle et al. 2011).

Figure 3 shows a comparison between the observed masses and radii of Kepler-16 A and B and the theoretical evolutionary models of Baraffe et al. (1998). Models are shown for 1 and 5 Gyr for a mixing-length parameter of 1.0. The 1 Gyr model 


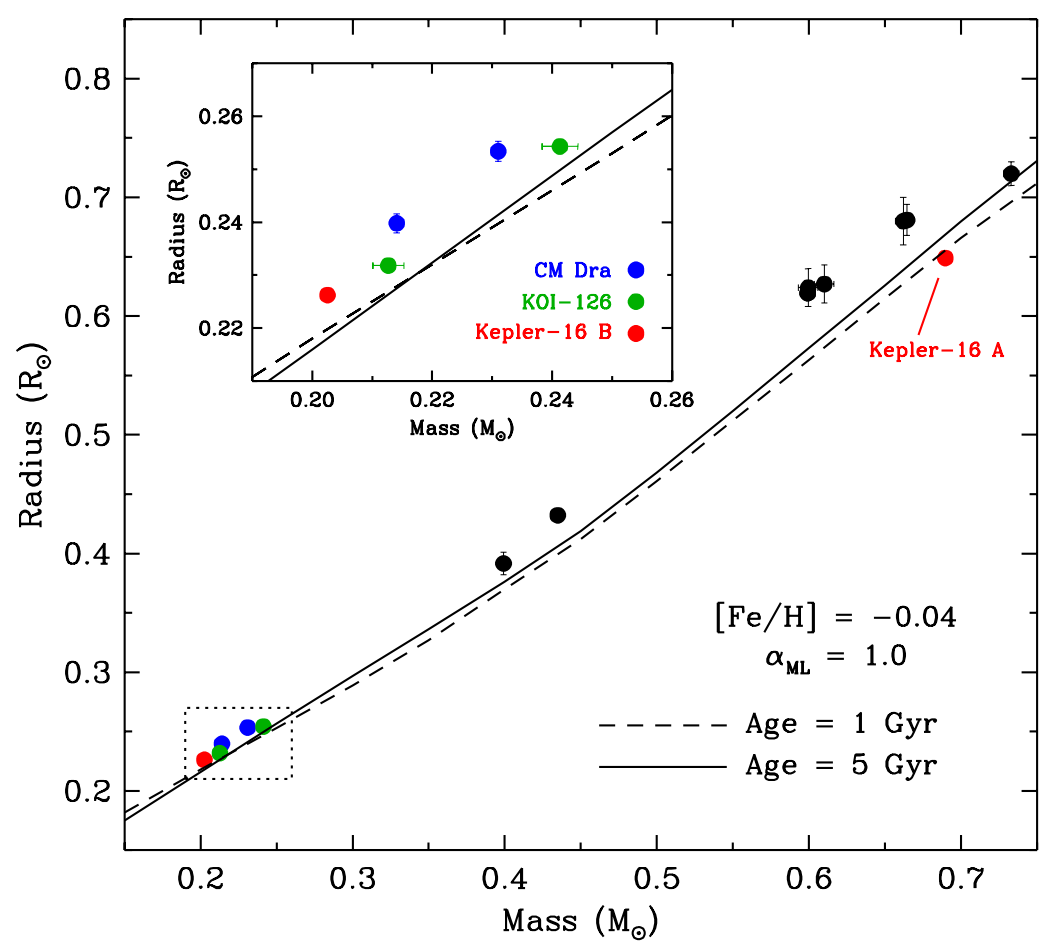

Figure 3. Theoretical and observed masses and radii of low-mass stars. The model curves are from Baraffe et al. (1998), for a metallicity of -0.04 (obtained by linear interpolation between 0.00 and -0.50 ) and a mixing-length parameter $\alpha_{\mathrm{ML}}=1.0$. In addition to Kepler-16 (Doyle et al. 2011), data are shown for other low-mass stars for which the masses and radii have been determined to within 3\% according to the rigorous criteria used by Torres et al. (2010). Those systems are KOI-126 (Carter et al. 2011), YY Gem (Torres \& Ribas 2002), CU Cnc (Ribas 2003), GU Boo (López-Morales \& Ribas 2005), NGC 2204 S892 (Rozyczka et al. 2009$)$, and IM Vir (Morales et al. 2009).

(A color version of this figure is available in the online journal.)

gives the best match to the primary mass and radius, although it is also possible to obtain a good fit at $5 \mathrm{Gyr}$ by increasing the mixing-length parameter toward the solar value of 1.9 (as might be expected for the primary; see, e.g., Demory et al. 2009). The models also match the measured effective temperature of the primary. Interestingly the calculated primary radius is within a few percent of the observed radius. This is in contrast to the other stars of similar mass shown in Figure 3, for which the observed radii are 10\%-15\% larger than the model predictions. Such discrepancies have been attributed to high activity and rapid rotation in the stars that have been studied closely (see, e.g., Chabrier et al. 2007). The secondary star's mass and radius are also within a few percent of the calculated values. We leave a more detailed comparison with models for future work, which should take into account the uncertainty in the metallicity as well as the $\alpha$-element abundances (which have not yet been measured).

\subsection{Formation Scenarios}

Binary stars are thought to form by fragmentation of collapsing prestellar cores, with close binaries such as Kepler-16 possibly resulting from fragmentation during a late, isothermal phase of the collapse (see, e.g., Goodwin et al. 2007). Stable hierarchial triples can also be produced by fragmentation (Boss 1991; Bate 2009), raising the question of whether the three bodies of Kepler- 16 formed in this manner.

In this scenario one would expect a tendency for the fragments to be aligned, but there is no obvious reason why the alignment would be as close as is observed for Kepler-16. Among the triple systems that formed in a large-scale simulation by Bate (2009), the orbital planes were typically misaligned by $\approx 60^{\circ}$. Furthermore, there are some close binaries with much larger obliquities than Kepler-16 A, such as DI Herculis (Albrecht et al. 2009).

This suggests an additional chapter is needed in the story, beyond fragmentation. Perhaps after their formation the stars continued to accrete substantially from a circumbinary disk, which would have decreased their orbital separation and aligned their spin axes (Bate et al. 2002). Or the primary could have formed with a massive circumstellar disk, which then fragmented to form the secondary. In either of these scenarios, the planet could have formed by core accretion near the inner edge of the circumbinary disk (see, e.g., Pierens \& Nelson 2008; Marzari et al. 2008).

\subsection{Evidence for Tidal Evolution}

Another process that could have reduced the stellar obliquity is tidal evolution. In the fullness of time, tides synchronize and align the spins of a binary system, and circularize their orbit (Zahn 1977; Hut 1981). Conventional wisdom would say that tides are irrelevant for Kepler-16 due to the relatively long orbital period (41 days) and wide separation ( $a=0.22 \mathrm{AU}$, $\left.a / R_{\star}=74\right)$, and indeed the orbital eccentricity of 0.16 shows that circularization is incomplete. On the other hand, synchronization and alignment should be faster than circularization because the rotational angular momentum is smaller than the orbital angular momentum. More broadly, tidal evolution timescales are poorly known, especially for spin evolution (see, e.g., Mazeh 2008).

An order-of-magnitude assessment of the importance of tidal evolution begins with the observation that most late-type binaries with periods $<10$ days have circular orbits (Mazeh 2008). Therefore, the circularization timescale is $\sim 5 \mathrm{Gyr}$ for $a / R_{\star} \sim 20$. If this timescale varies as $\left(a / R_{\star}\right)^{8}$ (Zahn 1977), 
then for Kepler- 16 it is $5(74 / 20)^{8} \sim 2 \times 10^{5}$ Gyr, i.e., consistent with the observation that the orbit is still eccentric. We further suppose that the spin evolution timescale is smaller by a factor of $\sim 10^{4}$ (the ratio of orbital to rotational angular momenta) and varies as $\left(a / R_{\star}\right)^{6}$ (Zahn 1977). Then the spin evolution timescale for Kepler-16 would be $5 \times 10^{-4}(74 / 20)^{6} \sim 1$ Gyr. This is corroborated by the more detailed tidal model of Terquem et al. (1998): using their Equations (41) and (42), the timescales for circularization and synchronization are $10^{4} \mathrm{Gyr}$ and $2 \mathrm{Gyr}$, respectively. These calculations are subject to the well-known uncertainties in tidal evolution timescales, but they do suggest that the low obliquity of Kepler-16 $\mathrm{A}$ is at least partly a consequence of tidal evolution.

In this context, the rotation period of the primary is intriguing. According to the tidal theory of Hut (1981), before the orbit circularizes the stellar spins evolve into a pseudosynchronous state, with spin periods shorter than the orbital period due to the enhanced tidal dissipation at pericenter passages. For Kepler16 A, using Equation (42) of Hut (1981) with $e=0.15944$ (Doyle et al. 2011), the predicted pseudosynchronous period is 35.62 days, in agreement with the measured period of $35.1 \pm 1.0$ days. As noted in Section 4.1, though, the measured rotation period is not unusual even for an isolated star, and the agreement with the pseudosynchronous value may be a coincidence. A more detailed study of the tidal evolution of this unique triple system is warranted, as are further spin-orbit studies of relatively wide binaries.

We thank Brice-Olivier Demory, Nevin Weinberg, and Jamie Lloyd for helpful discussions. Work by J.N.W. and S.A. was supported by NASA Origins award NNX09AB33G. G.T. acknowledges partial support from the NSF through grant AST-1007992. Funding for the Kepler Discovery mission is provided by NASA's Science Mission Directorate. The W. M. Keck Observatory is operated as a scientific partnership among the California Institute of Technology, the University of California, and the National Aeronautics and Space Administration, and was made possible by the generous financial support of the W. M. Keck Foundation. We extend special thanks to those of Hawaiian ancestry on whose sacred mountain of Mauna Kea we are privileged to be guests.

Facilities: Keck:I(HIRES), Kepler

\section{REFERENCES}

Albrecht, S., Reffert, S., Snellen, I. A. G., Quirrenbach, A., \& Mitchell, D. S. 2007, A\&A, 474, 565
Albrecht, S., Reffert, S., Snellen, I. A. G., \& Winn, J. N. 2009, Nature, 461, 373 Albrecht, S., Winn, J. N., Carter, J. A., Snellen, I. A. G., \& de Mooij, E. J. W. 2011, ApJ, 726, 68

Bailyn, C. D. 1989, ApJ, 341, 175

Baraffe, I., Chabrier, G., Allard, F., \& Hauschildt, P. H. 1998, A\&A, 337, 403

Barnes, S. A. 2007, ApJ, 669, 1167

Barnes, S. A. 2010, ApJ, 722, 222

Barnes, S. A., \& Kim, Y.-C. 2010, ApJ, 721, 675

Bate, M. R. 2009, MNRAS, 392, 590

Bate, M. R., Bonnell, I. A., \& Bromm, V. 2002, MNRAS, 336, 705

Borucki, W. J., Koch, D., Basri, G., et al. 2010, Science, 327, 977

Boss, A. P. 1991, Nature, 351, 298

Butler, R. P., Marcy, G. W., Williams, E., et al. 1996, PASP, 108, 500

Caldwell, D. A., Kolodziejczak, J. J., Van Cleve, J. E., et al. 2010, ApJ, 713, L92

Carter, J. A., Fabrycky, D. C., Ragozzine, D., et al. 2011, Science, 331, 562

Chabrier, G., Gallardo, J., \& Baraffe, I. 2007, A\&A, 472, L17

Claret, A. 2000, A\&A, 363, 1081

Demory, B.-O., Ségransan, D., Forveille, T., et al. 2009, A\&A, 505, 205

Doyle, L. R., Carter, J. A., Fabrycky, D. C., et al. 2011, Science, 333, 1602

Goodwin, S. P., Kroupa, P., Goodman, A., \& Burkert, A. 2007, in Protostars and Planets V, ed. B. Reipurth, D. Jewitt, \& K. Keil (Tucson, AZ: Univ. Arizona Press), 133

Gray, D. F. 2005, The Observation and Analysis of Stellar Photospheres (3rd ed.; Cambridge: Cambridge Univ. Press)

Hall, J. C., Henry, G. W., Lockwood, G. W., Skiff, B. A., \& Saar, S. H. 2009, AJ, 138,312

Hosokawa, Y. 1953, PASJ, 5, 88

Hut, P. 1981, A\&A, 99, 126

Ivanova, N. 2008, in Proc. ESO Workshop, Multiple Stars Across the H-R Diagram, ed. S. Hubrig, M. Petr-Gorzens, \& A. Tokovinin (Berlin: Springer), 101

Jenkins, J. M., Caldwell, D. A., Chandrasekaran, H., et al. 2010, ApJ, 713, L87

Koch, D. G., Borucki, W. J., Basri, G., et al. 2010, ApJ, 713, L79

López-Morales, M., \& Ribas, I. 2005, ApJ, 631, 1120

Mamajek, E. E., \& Hillenbrand, L. A. 2008, ApJ, 687, 1264

Marzari, F., Thébault, P., \& Scholl, H. 2008, ApJ, 681, 1599

Mazeh, T. 2008, in Tidal Effects in Stars, Planets and Disks, ed. M.-J. Goupil \& J.-P. Zahn (EAS-PS 29; Noordwijk: EAS), 1

McLaughlin, D. B. 1924, ApJ, 60, 22

Mikkola, S. 1984, MNRAS, 207, 115

Morales, J. C., Torres, G., Marschall, L. A., \& Brehm, W. 2009, ApJ, 707, 671

Noyes, R. W., Hartmann, L. W., Baliunas, S. L., Duncan, D. K., \& Vaughan, A. H. 1984, ApJ, 279, 763

Ohta, Y., Taruya, A., \& Suto, Y. 2005, ApJ, 622, 1118

Pierens, A., \& Nelson, R. P. 2008, A\&A, 483, 633

Ribas, I. 2003, A\&A, 398, 239

Rossiter, R. A. 1924, ApJ, 60, 15

Rozyczka, M., Kaluzny, J., Pietrukowicz, P., et al. 2009, Acta Astron., 59, 385

Shporer, A., \& Brown, T. 2011, ApJ, 733, 30

Sterzik, M. F., \& Tokovinin, A. A. 2002, A\&A, 384, 1030

Terquem, C., Papaloizou, J. C. B., Nelson, R. P., \& Lin, D. N. C. 1998, ApJ, 502, 788

Torres, G., Andersen, J., \& Giménez, A. 2010, A\&ARv, 18, 67

Torres, G., \& Ribas, I. 2002, ApJ, 567, 1140

Valenti, J. A., \& Piskunov, N. 1996, A\&AS, 118, 595

Zahn, J.-P. 1977, A\&A, 57, 383 